\title{
ERRATUM
}

\section{Nanogel antigenic protein-delivery system for adjuvant-free intranasal vaccines}

Tomonori Nochi, Yoshikazu Yuki, Haruko Takahashi, Shin-ichi Sawada, Mio Mejima, Tomoko Kohda, Norihiro Harada, II Gyu Kong, Ayuko Sato, Nobuhiro Kataoka, Daisuke Tokuhara, Shiho Kurokawa, Yuko Takahashi, Hideo Tsukada, Shunji Kozaki, Kazunari Akiyoshi and Hiroshi Kiyono

Nature Materials 9, 572-578 (2010); published online: 23 June 2010; corrected after print: 2 July 2010.

On the first page of the PDF and printed versions of this Letter originally published, the full list of authors and their affiliations should have been included as shown below:

Tomonori Nochi ${ }^{1+}$, Yoshikazu Yuki ${ }^{1 \dagger}$, Haruko Takahashi², Shin-ichi Sawada ${ }^{2}$, Mio Mejima ${ }^{1}$, Tomoko Kohda ${ }^{3}$, Norihiro Harada ${ }^{4}$, Il Gyu Kong ${ }^{1}$, Ayuko Sato ${ }^{1}$, Nobuhiro Kataoka ${ }^{1}$, Daisuke Tokuhara ${ }^{1}$, Shiho Kurokawa ${ }^{1}$, Yuko Takahashi ${ }^{1}$, Hideo Tsukada ${ }^{4}$, Shunji Kozaki ${ }^{3}$, Kazunari Akiyoshi ${ }^{2}$ and Hiroshi Kiyono ${ }^{1 *}$

${ }^{1}$ Division of Mucosal Immunology, Department of Microbiology and Immunology, The Institute of Medical Science, The University of Tokyo, Tokyo 108-8639, Japan, ${ }^{2}$ Department of Organic Materials, Institute of Biomaterials and Bioengineering, Tokyo Medical and Dental University, Tokyo 101-0062, Japan, ${ }^{3}$ Laboratory of Veterinary Epidemiology, Department of Veterinary Science, Graduate School of Life and Environmental Sciences, Osaka Prefecture University, Osaka 599-8531, Japan, ${ }^{4}$ PET Center, Central Research Laboratory, Hamamatsu Photonics K.K., Shizuoka 434-8601, Japan. ${ }^{\dagger}$ These authors contributed equally to this work. ${ }^{*}$ Present address: Division of Infectious Diseases, Center for AIDS Research, University of North Carolina, Chapel Hill, North Carolina 27599, USA. *e-mail: kiyono@ims.u-tokyo.ac.jp.

This has been corrected in the PDF version of this Letter. 